\title{
Vascular and Interventional Radiology in Sudan
}

\author{
Alaaeldeen Mohammed Mohammed ${ }^{1}$ \\ ${ }^{1}$ Medical Imaging Department, King Abdul-Aziz Medical City, Jeddah, \\ Saudi Arabia \\ Arab J Intervent Radiol 2021;5:68-70.
}

\begin{abstract}
Address for correspondence Alaaeldeen Mohammed Ahmed, MD Radiologist, Medical Imaging King Abdulaziz Medical City, Jeddah-KSA 21423 (e-mail: Alaaeldeen29rad@outlook.com).
\end{abstract}
Abstract
Keywords
- interventional
- radiology
- Sudan healthcare
- interventional radiology training

There are only 4 interventional radiologists working in Sudan with many difficulties such as lack of resources and high cost of interventional equipments and procedures. This result in shortage of interventional radiology services which is severely affecting the quality of patient care.

\section{Introduction}

Sudan is the third largest populated country in the Arab world after Egypt and Algeria with population of approximately 42 million. ${ }^{1}$ The World Health Organization (WHO) estimates the number of physicians in Sudan at 2.26 per 10,000 population, ${ }^{2}$ which is far less than the average number of other Arab and worldwide developed countries as shown in -Fig. 1. There is no official released data about the workforce of radiologists in Sudan. However, on personal connection and estimation, there are $\sim 550$ radiologists registered at Sudan Medical Council, and less than 300 radiologists and four vascular interventional radiologists (VIR) are currently practicing in the country.

\section{Radiology Training in Sudan}

The postgraduate medical training in Sudan is under Sudan Medical Specialization Board (SMSB), which is providing 33 specialties since its establishment in 1996. Radiology training in Sudan started at the University of Khartoum in 1992, but the program did not shift to be under SMSB regulations until 2003, when SMSB became the official governmental body responsible for the selection of candidates, training programs, and exams. $^{3}$ However, most of the training centers belong to private sector due to well-equipped radiology departments compared with the governmental healthcare institutions. Since the training started under the regulations of SMSB, approximately 250 physicians have finished their training and have been certified by the board. The process of radiology training starts with a selection exam that determines the candidates who will enter the 4-year training program, which consists of clinical radiological rotations in different hospitals. The assessment of trainees is based on continuous log book evaluation and two exams, one after finishing the first year and a final exam at the end of the fourth year.

The number of radiology trainees accepted is increasing yearly as it started by 12 trainees in 2003 and is currently 35 trainees in average. Such increase in number of trainees depends on the accreditation of new centers for training and maintaining the accreditations of the old ones.

\section{VIR Practice in Sudan}

Only four VIR are currently working in Sudan in the capital city of Sudan, Khartoum. As there is no interventional radiology training fellowship in Sudan, all the interventionists currently working have been trained either in Jordan or Malaysia. published online January 24, 2021
DOI https://doi.org/ 10.1055/s-0041-1742089. ISSN 2542-7075.

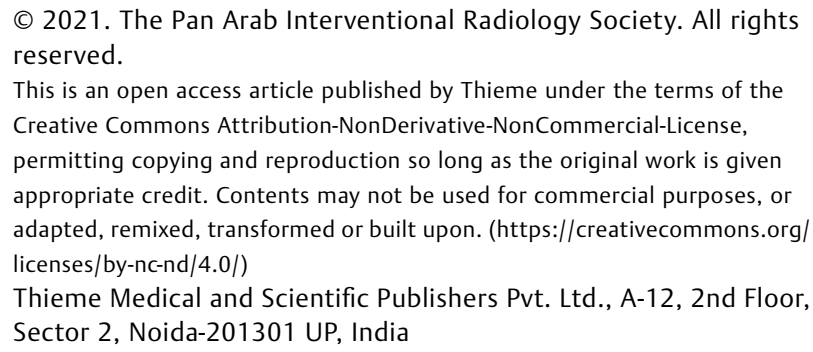

This is an open access article published by Thieme under the terms of the Creative Commons Attribution-NonDerivative-NonCommercial-License, permitting copying and reproduction so long as the original work is given appropriate credit. Contents may not be used for commercial purposes, or adapted, remixed, transformed or built upon. (https://creativecommons.org/ licenses/by-nc-nd/4.0/)

Thieme Medical and Scientific Publishers Pvt. Ltd., A-12, 2nd Floor, Sector 2, Noida-201301 UP, India 
60

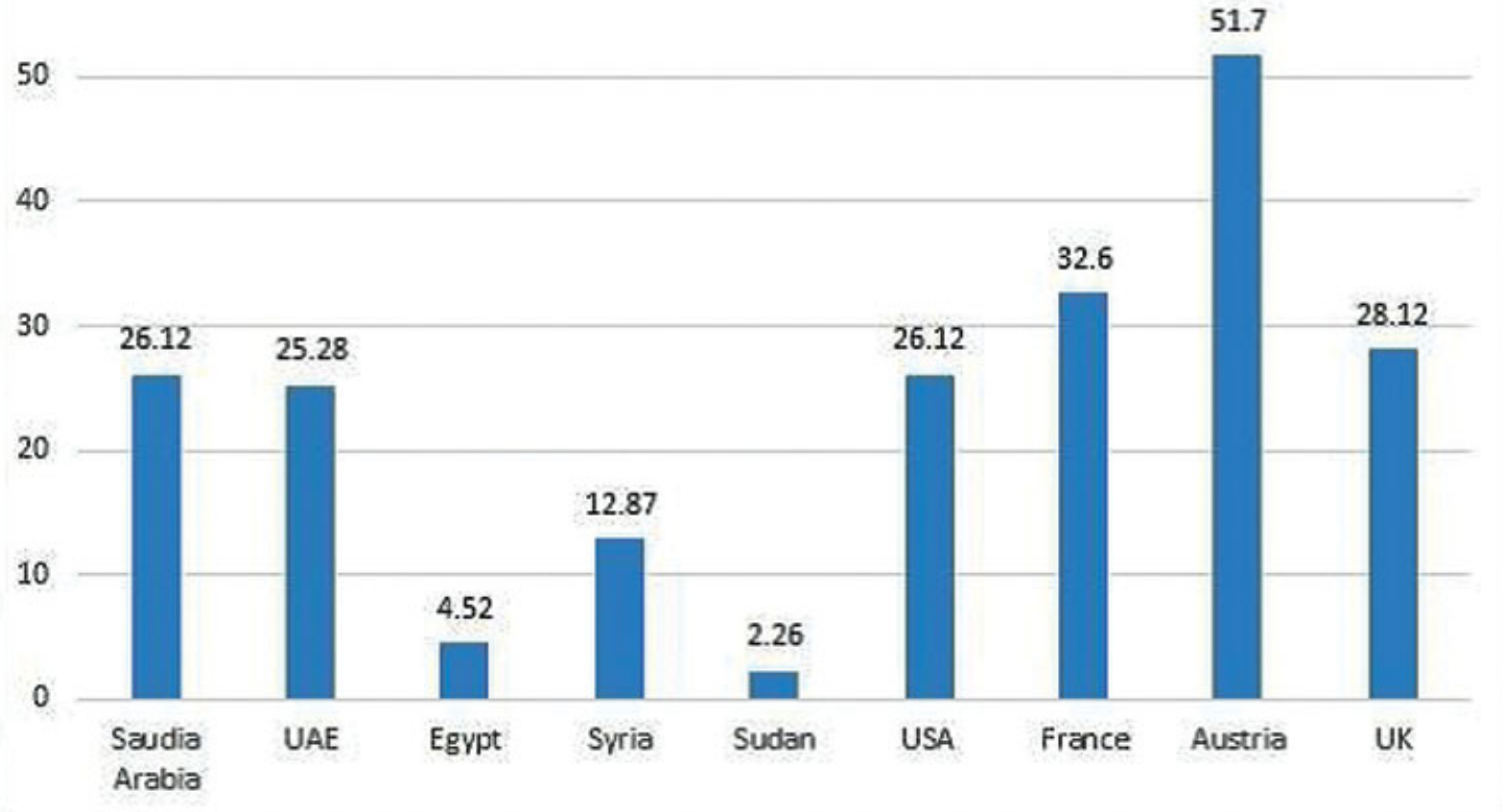

Fig. 1 Medical doctors per 100.000 population of some Arab and developed countries.

Most of the basic interventional procedures such as biopsies and drainage are performed by the diagnostic radiologists. Some other procedures like percutaneous insertion of nephrostomy tubes and tunneled dialysis catheters are performed by urologists and vascular surgeons, respectively. No dedicated IR suite is available for interventional procedures, which are performed either in fluoroscopic rooms or in shared angiography/cardiac catheterization suites that are available at least in five private hospitals. As a result, many patients lack the access to facilities supported by IR services.

\section{Obstacles}

There are several significant obstacles that must be overcome to safely and practically implement and sustain interventional radiology service in Sudan. These obstacles include infrastructure and relevant logistics, social and cultural obstacles. First, no dedicated VIR service is available in any governmental healthcare institutions and hospitals, which are essentially not equipped with enough imaging modalities due to funding issues. Second obstacle is the high cost of the VIR procedures that is very high in comparison to the average Sudanese income with no health insurance companies covering VIR procedures in their plans due to which both physicians and patients are forced to choose more available conventional, but more invasive surgical options. Third obstacle is the lack of professional awareness among differ- ent physicians about the advantages of VIR and its role in the management of various clinical conditions, for example, management of postlaparotomy active bleeding with another laparotomy with all the potential risks instead of a less invasive emergency embolization.

Fourth, the number of available trained VIRs is less than any other Arab country due to unavailable training program and immigration of radiologists to work abroad especially in the Gulf countries.

Although there is governmental fund for improving the department of radiology equipment including VIR in one of the biggest governmental hospitals, there is no available national plan for expanding VIR service in Sudan at all aspects.

\section{Future Plans}

All plans should focus on introducing strategies at governmental and private sector levels for implementing VIR service in the healthcare institutions and overcoming the obstacles. Such strategies should analyze the obstacles and introduce practical and innovative solutions that are customized to the Sudanese healthcare needs. They can be developed by healthcare governmental and academic bodies in addition to national medical societies to recognize, introduce, and incorporate VIR into clinical practice and healthcare services with assistance from the international humanitarian and medical organizations. This will be a significant and positive change in the patients care and quality of life. 
SMSB should recognize VIR as a specialty and should establish training programs in the equipped and staffed national hospitals in collaboration with the currently working VIR consultants in addition to increasing the awareness about VIR among the medical students and residents. Additionally, SMSB can seek opportunities to collaborate with international societies such Pan Arab Interventional Radiology Society (PAIRS), which is considered one of the most active regional VIR societies to establish and develop training and educational courses in VIR for Sudanese trainees.

In conclusion, incorporating VIR practice in Sudan healthcare is facing obstacles that require holistic approach at governmental and designated national medical authorities. This requires exploring potential opportunities and seeking collaboration with international organizations and regional medical societies especially PAIRS to introduce and incorporate VIR into the healthcare system.

\section{Conflicts of Interest}

None.

\section{Acknowledgment}

For prof Abdallah Jabir, head department of Sudan Diagnostic Radiology Board for his available guidance and support.

We would like to thank Dr. Azzam Khankan, Consultant Interventional Radiologist, NGHA-JEDDAH for his advice, help, and support.

This manuscript is not sent to any journals or presented in any conference or meetings.

\section{References}

1 Khalife L. UDC7RFYFNRhrH8pI. (2018, July 11). Arab countries ranked by population size. StepFeed. Accessed December 31, 2021 at: https:// stepfeed.com/arab-countries-ranked-by-population-size-7779

2 Medical doctors (per 10,000 population) [Internet]. World Health Organization. World Health Organization; [cited 2021Jan24]. Accessed December 20, 2021 from: https://www.who.int/data/ gho/data/indicators/indicator-details/GHO/medical-doctors(per-10-000-population)

3 Home [Internet]. Sudan Medical Specialization Board. [cited 2021Jan1]. Accessed December 20, 2021 from: https://smsb. gov.sd/en/ 